\title{
Repercusión neurológica por cambios en el flujo sanguíneo cerebral en neonatos sometidos a cirugía cardiovascular
}

\author{
Laura Carina Feria-Kaiser, ${ }^{1}$ Mario Enrique Rendón-Macías, ${ }^{2 *}$ Manuel Martínez-Meraz, ${ }^{3}$ \\ María del Rocío Méndez-Méndez' y Héctor Jaime González-Cabello' \\ ${ }^{1}$ Instituto Mexicano del Seguro Social, Centro Médico Nacional Siglo XXI, Hospital de Pediatría; ${ }^{2}$ Universidad Panamericana, Escuela de Medicina, \\ Departamento de Salud Pública; ${ }^{3}$ Instituto Politécnico Nacional, Escuela Superior de Medicina. Ciudad de México, México
}

\section{Resumen}

Introducción: La cirugía de cardiopatías congénitas puede generar alteraciones perfusorias cerebrales con repercusión neurológica. Objetivo: Analizar la relación del índice de resistencia (IR) vascular cerebral periquirúrgico con funciones neurológicas mediatas posteriores a cirugía de cardiopatía congénita. Método: Estudio de cohorte prospectivo de 34 neonatos en quienes se determinó IR de la arteria basilar, niveles séricos de oxígeno, dióxido de carbono y lactato, antes y después de procedimientos paliativos o correctivos. Relacionamos el IR prequirúrgico con la capacidad posquirúrgica para iniciar la vía enteral o restablecer la respiración espontánea no asistida. Resultados: Se integraron tres grupos: 17 neonatos con IR alto $>0.73$, cinco con IR normal de 0.63 a 0.73 y seis con IR bajo < 0.63 . En los primeros persistió IR alto en el posquirúrgico, con hiperlactatemia e hipoxia persistentes; en 86 \% no se logró iniciar la vía enteral ni retirar la ventilación asistida. En los segundos, el IR se mantuvo en valores normales. En los terceros, si bien el $I R$, el lactato sérico y la presión arterial de oxígeno tendieron a normalizarse, 71 \% presentó daño neurológico grave. Conclusiones: Los cambios en el IR fueron frecuentes, aunque el daño neurológico parece presentarse más cuando el IR se mantiene alto, posiblemente asociado a flujos cerebrales bajos.

PALABRAS CLAVE: Índice de resistencia. Cirugía cardiovascular. Cardiopatía congénita. Neonato.

\section{Neurological repercussions of changes in cerebral blood flow in neonates undergoing cardiovascular surgery}

\section{Abstract}

Introduction: Surgery for congenital heart disease can generate cerebral perfusion-associated alterations with neurological repercussions. Objective: To analyze the relationship of peri-surgical cerebrovascular resistance index (RI) with mediate neurological functions after congenital heart disease surgery. Method: Prospective cohort study of 34 neonates in whom basilar artery RI, serum oxygen, carbon dioxide and lactate levels were determined before and after palliative or corrective procedures. We related pre-surgical $R I$ with post-surgical ability to initiate the enteral route or to restore unassisted spontaneous breathing. Results: Three groups were formed: 17 neonates with high $R I$ (> 0.73), five with normal $R I$ (0.63-0.73) and six with low $R I(<0.63)$. In the former group, high $R I$ persisted in the postoperative period, with persistent hyperlactatemia and hypoxia; in $86 \%$, the enteral route could not be initiated, and neither could assisted ventilation be withdrawn. In the second group, IR remained within normal values. In the third group, although $R I$, serum lactate and arterial oxygen pressure tended to normalize, $71 \%$ had severe neurological damage. Conclusions: $R$ l changes were common, although neurological damage appears to occur more commonly when $\mathrm{Rl}$ remains high, possibly associated with low cerebral blood flow.

KEY WORDS: Resistance index. Cardiovascular surgery. Congenital heart disease. Neonate.

Correspondencia:

*Mario Enrique Rendón-Macías

E-mail: drmariorendon@gmail.com

DOI: 10.24875/GMM.19005491
Gac Med Mex. 2020;156:110-117

Disponible en PubMed

www.gacetamedicademexico.com

CC BY-NC-ND (http://creativecommons.org/licenses/by-nc-nd/4.0/). 


\section{Introducción}

El manejo terapéutico, ya sea paliativo o correctivo, de los neonatos con cardiopatía congénita compleja (CCC) es un reto por la alta mortalidad y las secuelas neurológicas. ${ }^{1,2}$ Las últimas se atribuyen a eventos hipóxico-isquémicos asociados con variaciones en el flujo sanguíneo cerebral. Si bien el daño neurológico pudiera iniciarse desde la vida fetal, ${ }^{3,4}$ el mayor riesgo suele presentarse después del nacimiento, antes o durante procedimientos correctivos o paliativos de la cardiopatía. ${ }^{5}$ Previo a este momento, el daño neurológico puede ser causado por hipoxia crónica propia de la CCC, eventos recurrentes o persistentes de hipercapnia o medicamentos preoperatorios; ${ }^{6-9}$ durante la cirugía cardiovascular puede ser ocasionado por el uso de circulación extracorpórea. ${ }^{10,11}$

Un determinante contra el daño hipóxico-isquémico es la conservación del flujo sanguíneo cerebral por autorregulación de las demandas metabólicas neuronales. ${ }^{12}$ La hipoxia prequirúrgica y las variaciones del volumen sanguíneo durante una cirugía pueden estropear estos mecanismos y facilitar daño neurológico. ${ }^{13}$ Un indicador indirecto de la funcionalidad de la autorregulación del flujo sanguíneo cerebral es el índice de resistencia vascular (IR),${ }^{14}$ el cual es medido por ultrasonido Doppler transfontanelar en arterias cerebrales; de estas, la arteria basilar proporciona más información y permite evaluar el efecto de la perfusión en las áreas básicas de supervivencia. ${ }^{15}$

Se espera que un neonato con CCC sin repercusión hemodinámica grave mantenga IR entre 0.63 y $0.73,{ }^{16}$ lo que no sucede si la malformación no es compensatoria. En neonatos con antecedentes de asfixia moderada o severa al nacer, el IR alterado ha sido asociado a daño neurológico. En neonatos con IR < 0.55 entre las 36 y 72 horas después del nacimiento se ha observado mayor deterioro neurológico posterior. ${ }^{14}$ Lo anterior no ha sido analizado en neonatos con CCC sometidos a cirugía cardiovascular. El objetivo de este trabajo fue evaluar el efecto de la cirugía cardiovascular en el IR, así como analizar si estos cambios afectan la funcionalidad neurológica temprana a cinco días del posoperatorio.

\section{Método}

Previa aprobación del Comité de Investigación y Ética local con registro R 2015-3603-32 y consentimiento informado de los padres de los niños, se reclutó a 34 pacientes atendidos de octubre de 2015 a mayo de 2016 en el Hospital de Pediatría del Centro Médico Nacional Siglo XXI, Instituto Mexicano del Seguro Social, Ciudad de México. Se incluyeron neonatos (< 28 días de vida) con CCC programados para cirugía cardiovascular paliativa o correctiva. Se excluyó a portadores de malformaciones múltiples extracardiacas, niños programados para cateterismo terapéutico o sin registro ultrasonográfico previo a la cirugía. Se eliminó del análisis final a los niños fallecidos durante la cirugía.

Los tratamientos médicos prequirúrgicos y la cirugía programada fueron decididos por un grupo de neonatólogos, cardiólogos y cirujanos cardiovasculares, si bien la decisión quirúrgica final fue determinada conforme los hallazgos transoperatorios. El manejo posquirúrgico fue independiente del resultado de los estudios ultrasonográficos y se basó en el consenso de los médicos responsables del niño.

Con ecografía Doppler pulsátil (Hewlett Packard modelo Sonos $5500^{\circledR}$ con transductor de $8 \mathrm{MHz}$ ) se realizaron cortes coronales cerebrales en la fontanela anterior hasta localizar el flujo de la arteria basilar. El IR se obtuvo con la fórmula (sístole - diástole)/ sístole, para lo cual se midió la onda de pulso más aceptable y se obtuvo la velocidad del pico sistólico máximo y la velocidad del pico diastólico final. El valor final consistió en el promedio de tres mediciones realizadas en 30 segundos. Las mediciones siempre fueron llevadas a cabo por un solo evaluador capacitado y en un periodo no mayor a 45 minutos antes del inicio de la cirugía. Se efectuaron dos mediciones posteriores, la primera al finalizar la cirugía y con al menos 30 minutos de estabilidad hemodinámica durante la estancia en terapia intensiva; la segunda, a las 18 horas. Además, en cada momento se determinó la presión arterial de oxígeno $\left(\mathrm{pO}_{2}\right)$, la presión arterial de dióxido de carbono $\left(\mathrm{pCO}_{2}\right)$ y el lactato sérico ( $\mathrm{mmol} / \mathrm{L})$.

De cada paciente se obtuvo edad gestacional, peso al nacimiento, tipo de cardiopatía, edad al procedimiento, peso prequirúrgico, puntuación $\mathrm{RASCH}-1,{ }^{17}$ uso de analgesia o sedación precarga, asistencia ventilatoria y alimentación enteral. En cuanto al procedimiento realizado se registró tiempo de circulación extracorpórea, tiempo de pinzamiento vascular y cierre diferido o no diferido del esternón. ${ }^{18}$

Para evaluar la condición funcional neurológica posterior a la cirugía, se registró si esta permitió o no el retiro de la asistencia ventilatoria y/o el inicio de la 


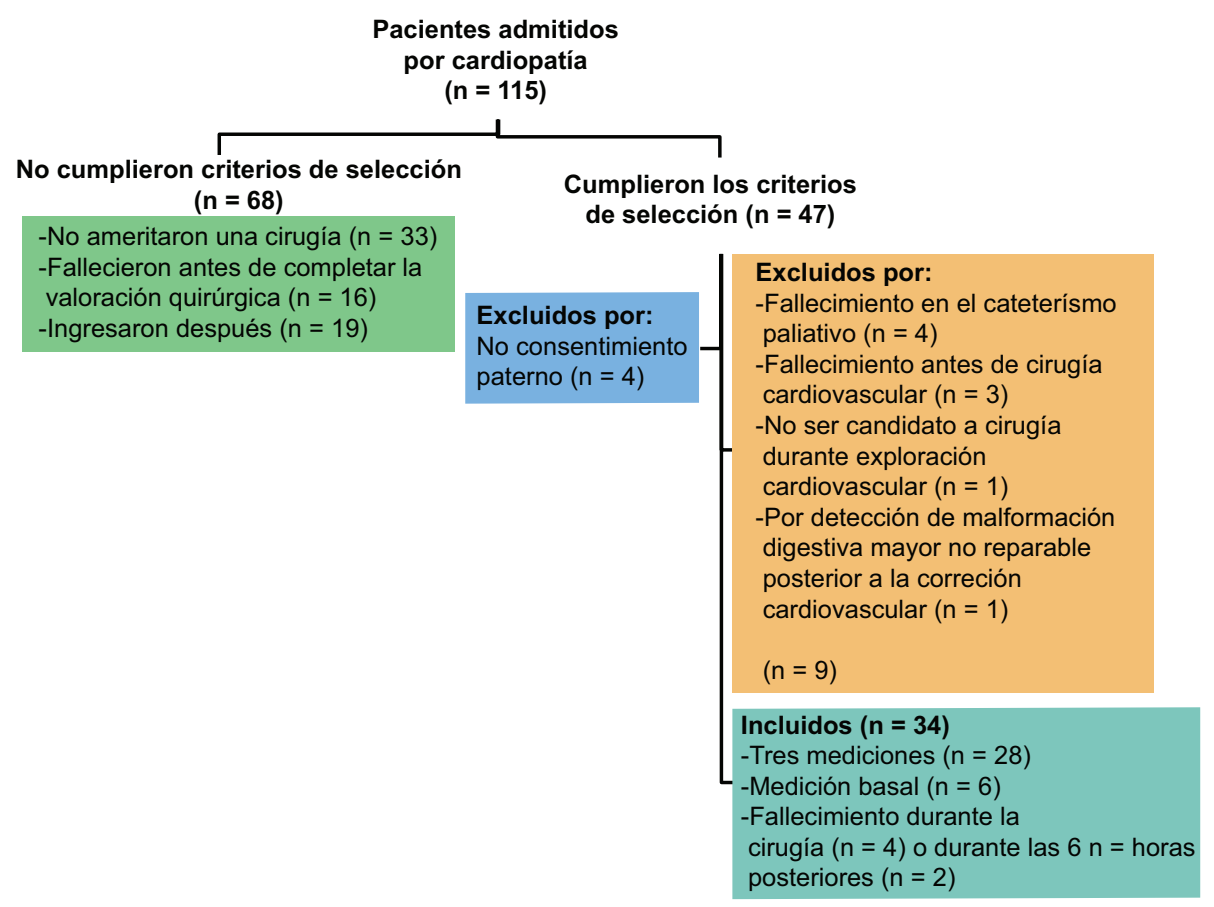

Figura 1. Flujograma de la selección de los neonatos con cardiopatía congénita compleja incluidos en el estudio.

alimentación durante los cinco días posteriores a la cirugía. La decisión del retiro de la ventilación asistida e inicio de la alimentación fueron determinadas por el equipo médico con base en criterios clínicos (automatismo respiratorio, equilibrio ácido-base, abdomen blando con peristalsis y evidencia de tránsito intestinal), independientemente del reporte del IR y de los investigadores. Finalmente, se determinó la supervivencia a este internamiento.

Los pacientes fueron agrupados conforme el IR prequirúrgico: normal, 0.63 a 0.73 ; bajo, < 0.63 ; alto, $>0.73$. Se obtuvieron frecuencias simples y porcentajes para variables categóricas y medianas con rangos intercuartílicos para las variables cuantitativas, dada su distribución anormal. Para determinar diferencias en los resultados en los tres momentos considerados se utilizó prueba no paramétrica de Wilcoxon o chi cuadrada. Todos los análisis se realizaron con el programa estadístico SPSS versión 24. Un valor de $p<0.05$ se consideró con significación estadística.

\section{Resultados}

Como se muestra en la Figura 1, fueron admitidos 115 pacientes con CCC, de los cuales 51 (42.8\%) cumplieron los criterios de selección; de estos, en 34 se obtuvieron mediciones prequirúrgicas y solo en 28 se lograron las tres.

\section{Características demográficas y clínicas precirugía}

Los datos se resumen en la Tabla 1. La mayoría de los neonatos fue del sexo masculino y a término, con una mediana de peso de $2900 \mathrm{~g}$ y 10 días de edad a la cirugía. La cardiopatía más frecuente consistió en transposición de grandes arterias, seguida de conexión anómala de los vasos pulmonares. En nueve, la cirugía fue paliativa y en el resto, correctiva; el cambio arterial (switch arterial) fue la más común (12/34; $35.2 \%) ; 75 \%$ de los neonatos recibió ventilación asistida, $50 \%$ medicación sedativa y $67 \%$ estaba en ayuno. El riesgo quirúrgico ajustado (RASCH-1) varió de 1 a 6, 61.7\% calificó con una puntuación de 3 .

De acuerdo con el IR prequirúrgico, siete pacientes $(20.5 \%)$ presentaron cifras normales, ocho $(23.5 \%)$ bajas y $19(55.8 \%)$ altas.

\section{Evolución poscirugía}

Debido a su defunción, seis pacientes no fueron medidos en los tres momentos programados: cuatro (11.7\%) fallecieron durante la circulación extracorpórea (dos con IR alto, uno con IR normal y uno con IR bajo) y dos (5.8\%) en el posoperatorio inmediato, ambos con IR bajo, durante la primera hora de salida de la circulación extracorpórea. 
Tabla 1. Datos clínicos y demográficos de 34 neonatos con cardiopatía congénita compleja

\begin{tabular}{|c|c|c|}
\hline Variable & Mediana & Cuartiles 1, 3 \\
\hline \multirow[t]{2}{*}{ Edad a la cirugía (días) } & 10 & 2,44 \\
\hline & Mediana & Min.-máx. \\
\hline \multirow[t]{2}{*}{ Peso en gramos a la cirugía } & 2900 & $1430-3835$ \\
\hline & $\mathbf{n}$ & $\%$ \\
\hline Sexo masculino & 22 & 64.7 \\
\hline $\begin{array}{l}\text { Edad gestacional al nacimiento } \\
\quad<37 \text { semanas } \\
\geq 37 \text { semanas }\end{array}$ & $\begin{array}{c}4 \\
30\end{array}$ & $\begin{array}{l}11.8 \\
88.3\end{array}$ \\
\hline $\begin{array}{l}\text { Tipo de anomalía cardiovascular } \\
\text { Transposición de grandes arterias } \\
\text { TGA + coartación aórtica/hipoplasia } \\
\text { Conexión anómala de venas } \\
\text { pulmonares } \\
\text { Interrupción aórtica/coartación } \\
\text { Síndrome de ventrículo izquierdo } \\
\text { hipoplásico } \\
\text { Atresia pulmonar } \\
\text { Aurícula y ventrículo únicos } \\
\text { Anomalía de Ebstein } \\
\text { Canal auriculoventricular }\end{array}$ & $\begin{array}{l}11 \\
2 \\
9 \\
3 \\
3 \\
\\
3 \\
1 \\
1 \\
1\end{array}$ & $\begin{array}{c}32.4 \\
5.9 \\
26.5 \\
\\
8.8 \\
8.8 \\
\\
8.8 \\
2.9 \\
2.9 \\
2.9\end{array}$ \\
\hline $\begin{array}{l}\text { Manejo prequirúrgico } \\
\text { Analgesia } \\
\text { Sedación } \\
\text { Asistencia ventilatoria } \\
\text { Alimentación enteral }\end{array}$ & $\begin{array}{c}8 \\
17 \\
25 \\
12\end{array}$ & $\begin{array}{l}23.5 \\
50.0 \\
73.5 \\
35.3\end{array}$ \\
\hline $\begin{array}{l}\text { Cirugías realizadas } \\
\text { Switch arterial } \\
\text { Fístula sistémica pulmonar } \\
\text { Reparación total de vasos } \\
\text { pulmonares } \\
\text { Plastia aórtica } \\
\text { Operación de Norwood } \\
\text { Vendaje arteria } \\
\text { pulmonar + reparación aórtica } \\
\text { Vendaje arteria pulmonar } \\
\text { RTVP + RPA }\end{array}$ & $\begin{array}{c}12 \\
8 \\
7 \\
\\
2 \\
2 \\
\\
1 \\
1 \\
1\end{array}$ & $\begin{array}{l}35.3 \\
23.5 \\
20.5 \\
\\
5.9 \\
5.9 \\
\\
2.9 \\
2.9 \\
2.9\end{array}$ \\
\hline $\begin{array}{l}\text { Puntuación RASCH-1 } \\
1 \\
3 \\
4 \\
6\end{array}$ & $\begin{array}{c}1 \\
21 \\
10 \\
2\end{array}$ & $\begin{array}{c}2.9 \\
61.8 \\
29.4 \\
5.9\end{array}$ \\
\hline $\begin{array}{l}\text { Tiempo de circulación extracorpórea } \\
<90 \text { minutos } \\
\geq 90 \text { minutos }\end{array}$ & $\begin{array}{c}3 \\
18\end{array}$ & $\begin{array}{c}8.0 \\
52.9\end{array}$ \\
\hline $\begin{array}{l}\text { Tiempo de pinzamiento } \\
<60 \text { minutos } \\
\geq 60 \text { minutos }\end{array}$ & $\begin{array}{c}8 \\
13\end{array}$ & $\begin{array}{l}23.5 \\
38.2\end{array}$ \\
\hline Cierre esternal diferido & 21 & 61.8 \\
\hline
\end{tabular}

TGA = transposición de grandes arterias, RTVP = reparación total de vasos pulmonares, RPA = reparación aórtica.
Requirieron cierre diferido del esternón 21 (61\%) pacientes supervivientes al procedimiento quirúrgico (Tabla 1). Dos pacientes fueron operados en dos ocasiones: en uno se realizó cerclaje de la arterial pulmonar inicial y switch arterial correctivo y en el otro, plastia de la aorta inicial y switch arterial posterior (Figura 1).

\section{Evolución del índice de resistencia poscirugía}

En la Figura 2 se muestra el IR posquirúrgico por grupos. En los pacientes con IR normal no se registraron diferencias entre sus valores iniciales (30 minutos) y 18 horas después ( $p=0.223$ ), además, en todo momento las cifras se mantuvieron entre 0.63 y 0.73 . En los niños con IR prequirúrgico bajo, este se normalizó en el posoperatorio: 0.65 a los 30 minutos y 0.68 a las 18 horas (prueba de Wilcoxon $p=0.09$ ). En los neonatos con IR prequirúrgico alto, este se mantuvo elevado: 0.82 a los 30 minutos y 0.81 a las 18 horas, $p=0.204$.

\section{Modificaciones en el $\mathrm{O}_{2}, \mathrm{CO}_{2}$ y lactato sérico en el periodo posqúirgico}

En todos los niños se observó incremento de la saturación de oxígeno después de la cirugía cardiovascular. El incremento fue más notorio y constante en el grupo con IR prequirúrgico bajo: 35,45 y $65 \mathrm{~mm} \mathrm{Hg}$ en las mediciones basal, a los 30 minutos y a las 18 horas ( $p=0.007)$. El cambio fue mínimo en los neonatos con IR normal: 40,61 y $57 \mathrm{~mm} \mathrm{Hg}$ en esas mismas mediciones ( $p=0.738)$, al igual que en aquellos con IR alto: 30, 46 y $50 \mathrm{~mm} \mathrm{Hg}(p=0.014)$ (Figura 3).

La presión parcial de dióxido de carbono en los neonatos con IR bajo y alto se redujo a los 30 minutos después de la cirugía y se normalizó a las 18 horas: en los niños con IR bajo, las mediciones basal, a los 30 minutos y a las 18 horas fueron de 36,32 y $35 \mathrm{~mm} \mathrm{Hg}(p=0.692)$; en aquellos con IR alto, de 36,32 y $36 \mathrm{~mm} \mathrm{Hg}$, respectivamente $(p=0.19)$. El grupo con IR normal no mostró cambios: basal de $36 \mathrm{~mm} \mathrm{Hg}$ y a los 30 minutos y 18 horas de $41 \mathrm{~mm} \mathrm{Hg}$ $(p=0.26)$ (Figura 4).

El lactato sérico estuvo elevado desde la medición prequirúrgica en todos los pacientes. En aquellos con IR normal no cambió durante el posoperatorio: $3 \mathrm{mmol} / \mathrm{L}$ en las mediciones prequirúrgica y a los 


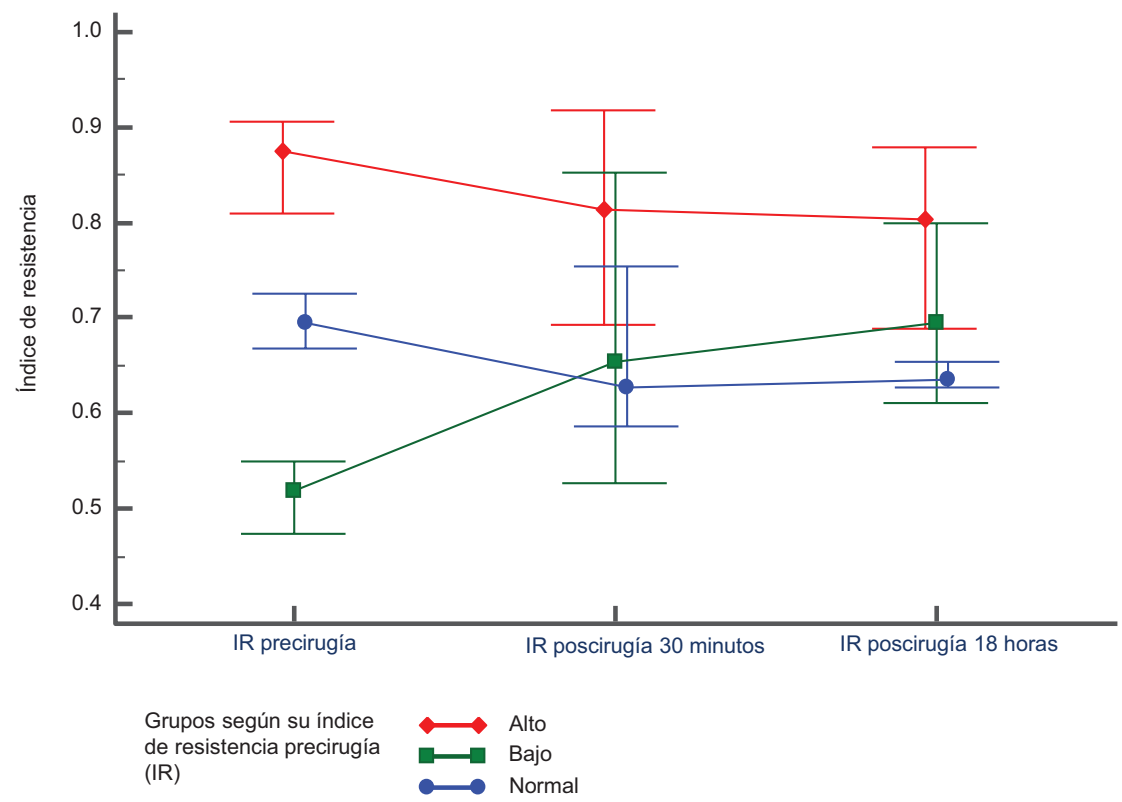

Figura 2. Cambios en los índices de resistencia (IR) después de la cirugía (30 minutos y 18 horas) según los valores prequirúrgicos (IR normal, $n=5$; IR bajo, $n=6$; IR alto, $n=17$ ). Los cuadrados, círculos y diamantes corresponden a las medianas y los bigotes, al rango intercuartílico 1-3. Con la prueba de Wilcoxon se obtuvo $p=0.091$ para IR bajo, $p=0.223$ para IR normal y $p=0.204$ para IR alto.

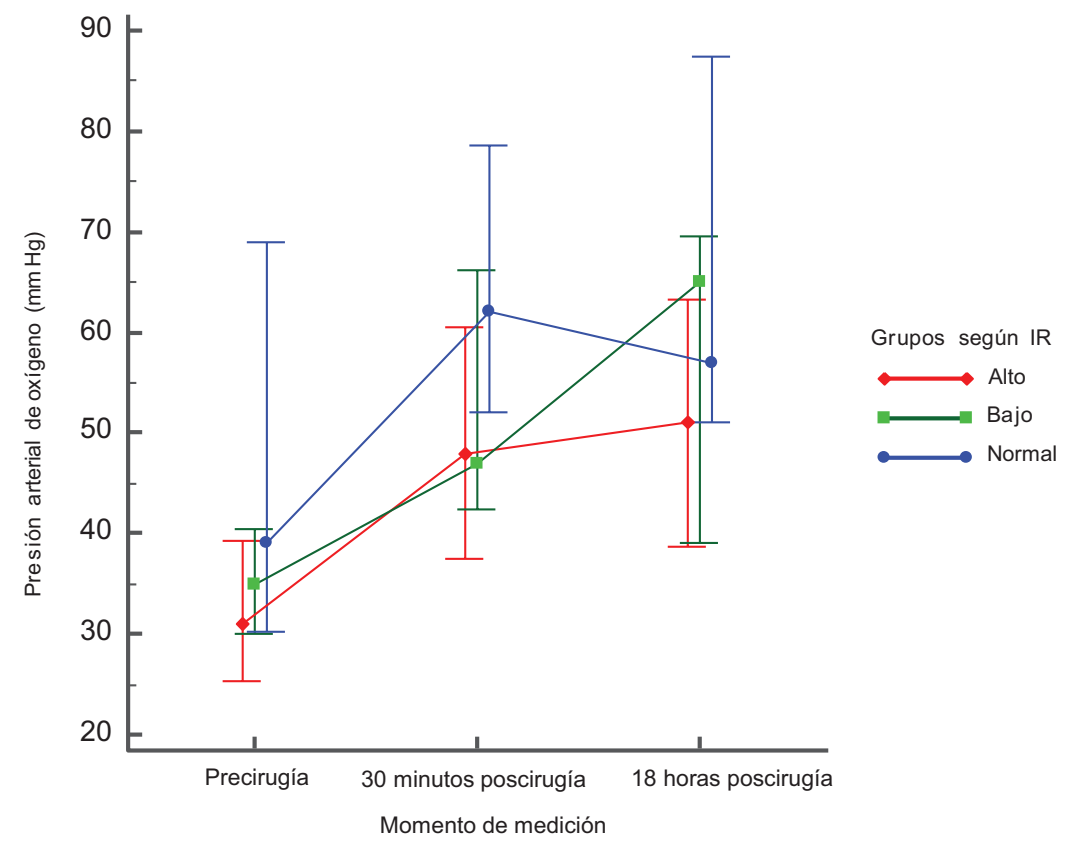

Figura 3. Niveles de presión arterial de oxígeno antes y después del procedimiento quirúrgico, según el índice de resistencia: normal, $n=5$; bajo, $n=6$; alto, $n=17$. Los cuadrados, círculos y diamantes corresponden a las medianas y los "bigotes", al rango intercuartílico 1-3. Con la prueba de Wilcoxon se obtuvo $p=0.007$ para IR bajo, $p=0.74$ para IR normal y $p=0.01$ para IR alto.

30 minutos y $2.5 \mathrm{mmol} / \mathrm{L}$ a las 18 horas $(p=0.554)$. En los pacientes con IR bajo se incrementó a los 30 minutos (2 a $4 \mathrm{mmol} / \mathrm{L})$, para mantenerse elevado a las 18 horas $(4 \mathrm{mmol} / \mathrm{L}, \mathrm{p}=0.247)$. En el grupo con IR alto se incrementó de 2.5 a $5.5 \mathrm{mmol} / \mathrm{L}$ a los 30 minutos; a las 18 horas bajó a valores cercanos a los prequirúrgicos $(3.5 \mathrm{mmol} / \mathrm{L}, \mathrm{p}<0.0001)$.

\section{Evolución clínica poscirugía}

La funcionalidad neurológica posquirúrgica fue evaluada en 28 pacientes (82.3\%). En $60.7 \%$ (17/28) fue desfavorable y no permitió iniciar la alimentación enteral ni el retiro de la ventilación asistida en los primeros cinco días posquirúrgicos (Tabla 2). No se 


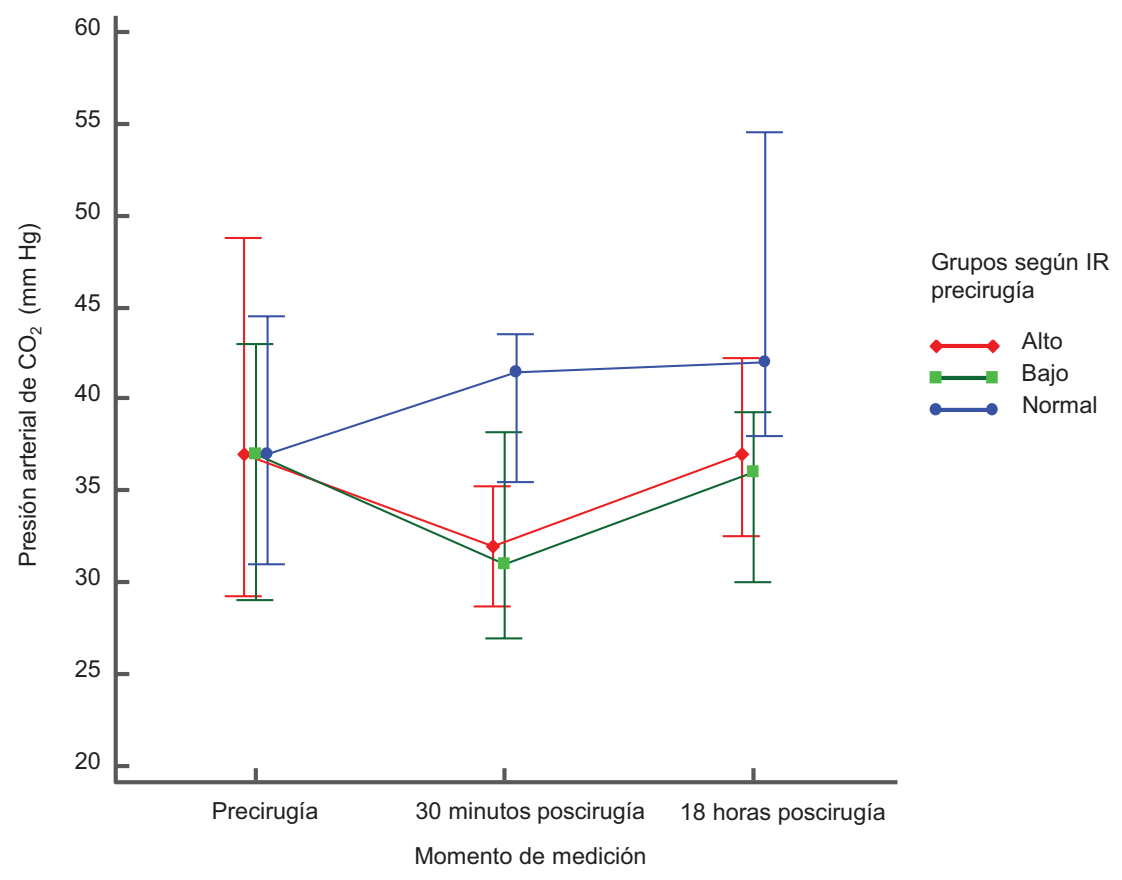

Figura 4. Niveles de presión arterial de dióxido de carbono antes y después del procedimiento quirúrgico, según el índice de resistencia (normal, $n=5$; bajo, $n=6$; alto, $n=17$ ). Los cuadrados, círculos y diamantes corresponden a las medianas y los "bigotes" al rango intercuartílico 1-3. Con la prueba de Wilcoxon se obtuvo $p=0.69$ para IR bajo, $p=0.26$ para IR normal y $p=0.13$ para IR alto.

Tabla 2. Capacidades neurológicas poscirugía según el índice de resistencia prequirúrgica en 28 neonatos con cardiopatía congénita compleja

\begin{tabular}{|c|c|c|c|c|c|c|c|c|}
\hline \multirow[t]{2}{*}{ Capacidad neurológica } & \multicolumn{2}{|c|}{$\begin{array}{l}\text { IR normal } \\
\qquad(n=6)\end{array}$} & \multicolumn{2}{|c|}{$\begin{array}{r}\text { IR bajo } \\
(n=5)\end{array}$} & \multicolumn{2}{|c|}{$\begin{array}{l}\text { IR alto } \\
(n=17)\end{array}$} & \multirow{2}{*}{$\frac{\begin{array}{c}\text { Total } \\
(\mathrm{n}=28)\end{array}}{\mathrm{n}}$} & \multirow[t]{2}{*}{$\mathbf{p}^{*}$} \\
\hline & n & $\%$ & n & $\%$ & n & $\%$ & & \\
\hline No permitió iniciar la alimentación enteral ni la respiración espontánea & 4 & 57.1 & 4 & 50 & 9 & 47.3 & 17 & 0.35 \\
\hline Solo se logró alimentación enteral o respiración espontánea & 2 & 33.3 & 1 & 20 & 4 & 23.5 & 7 & \\
\hline Se logró alimentación enteral y respiración espontánea & 0 & 0 & 0 & 0 & 4 & 23.5 & 4 & \\
\hline Muerte & 2 & 28.5 & 4 & 50 & 5 & 26.3 & 11 & \\
\hline
\end{tabular}

$\mathrm{IR}=$ índice de resistencia vascular. IR normal, 0.63-0.73; IR bajo, $\leq 0.63 ; \mathrm{IR}$ alto, $\geq 0.73$.

${ }^{*}$ Prueba de Pearson, coeficiente de contingencia $=0.34$.

pudo demostrar una diferencia estadísticamente significativa entre los grupos. Solo cuatro pacientes del grupo con IR alto (23.5 \%) lograron funcionalidad neurológica completa.

\section{Discusión}

Un primer hallazgo fue el IR bajo o alto en la mayoría de los pacientes $(79 \%)$ antes de la cirugía, lo que implica alta frecuencia de neonatos con flujo sanguíneo cerebral alterado posiblemente por cambios hemodinámicos desde la etapa fetal y relacionados con las cardiopatías..$^{19}$ Es posible que con la cirugía cardiovascular se redistribuyan los flujos y con ello el IR, como sucedió en la mayoría de los pacientes, cambie a niveles normales en las 18 horas posteriores a la cirugía. Cuando el flujo sanguíneo cerebral no se normaliza, potencialmente puede haber daño neurológico. ${ }^{20}$ Por otro lado, debido a la disposición de tres grupos diferentes según el IR prequirúrgico, fue posible evaluar patrones diferenciales en el comportamiento vasculocerebral secundario al reto hemodinámico que implica una cirugía compleja. En los neonatos con IR prequirúrgico normal se observó respuesta vasomotora cerebral conservada, con discreto aumento en el IR al final de la intervención quirúrgica, posiblemente por cambios de volumen por la reperfusión posterior al retiro de la circulación extracorpórea, ${ }^{11}$ pero que retornó al valor normal una vez estabilizado el paciente a las 18 horas. 
En el grupo con un IR prequirúrgico alto, ocho de los 19 pacientes tenían trasposición de grandes arterias o falla cardiaca derecha e hipóxica crónica, condiciones inductoras de incremento del IR; en esos pacientes, a pesar de una corrección del defecto con incremento de la presión parcial de oxígeno, no hubo vasodilatación consecuente con reducción sustancial del IR, lo cual puede explicarse por alteraciones en la saturación regional de oxígeno. ${ }^{20}$

Contrario a nuestra hipótesis de que el posoperatorio inmediato sería favorable en los niños con normalización del IR, esto solo sucedió en un paciente con IR previamente alto. Otros tres neonatos con IR alto precirugía también tuvieron un posoperatorio favorable, pero las cifras del IR continuaron siendo altas. En el resto se dificultó la decanulación o el inicio de la vía enteral o ambos.

Por el reducido número de pacientes y la interacción de múliples factores, principalmente los tipos de cardiopatía, fue difícil analizar las condiciones que afectaron la evolución neurológica. Particularmente en los neonatos con IR bajo, aunque se incrementaron los valores durante el posoperatorio, la evolución posterior fue muy mala: tres fallecieron y los otros no lograron autonomía respiratoria y enteral. Estos hallazgos son compatibles con los observados por Ilves $^{21}$ en pacientes con asfixia severa y explicados como daño por vasoparálisis secundaria a hiperperfusión retrasada. Quizás en los pacientes con IR alto, la autorregulación cerebral mantenía una mejor compensación a los cambios en la presión de la perfusión cerebral. ${ }^{15}$ Cuando esta autorregulación se daña, la eficacia depende exclusivamente de factores externos como la presión arterial y el volumen sanguíneo, entre otros. No dudamos que estos mecanismos se hubieran extinguido en los pacientes, a lo que contribuyeron las condiciones del tratamiento quirúrgico y anestésico. ${ }^{22}$

Otro factor adyuvante en varios pacientes fue el cierre diferido de esternón. Durante la espera, los pacientes estuvieron bajo sedación, analgesia e intubación, condiciones de alto riesgo para nuevos episodios hipóxico-isquémicos.

Deben ser consideradas dos limitaciones importantes de este estudio, una es el tamaño de muestra y la segunda, el tiempo de seguimiento. Si bien es difícil reproducir el estudio por las características de los pacientes, será necesario un estudio multicéntrico. Asimismo, se ha sugerido la resonancia magnética nuclear vascular como otra opción para la valoración periquirúrgica ${ }^{23} y$ analizar otros factores involucrados. ${ }^{24}$

\section{Conclusiones}

Una alta proporción de pacientes con CCC muestra alteración del IR cerebral antes del procedimiento cardiovascular y aunque la cirugía cardiovascular parece favorecer la normalización del IR, la repercusión neurológica pudiera ya estar determinada. En la mayoría de los pacientes fue difícil retirar la asistencia ventilatoria o iniciar la alimentación enteral debido al inadecuado control neurológico central.

\section{Agradecimientos}

Nuestro agradecimiento y reconocimiento a José Agustín Mercado Arellano, Rodolfo Rivas Ruiz, Juan O. Talavera, Marcela Pérez Rodríguez, Jorge Salmerón Castro y María Esther Ocharán Hernández.

\section{Referencias}

1. Hogue CW Gottesman R, Stearns J. Mechanisms of cerebral injury from cardiac surgery. Crit Care Clin. 2008;24:83-98.

2. Ortinau C, Beca J, Lambert J, Ferdman B, Alexopoulos D, Shimony JS, et al. Regional alterations in cerebral growth exist pre-operatively in infants with congenital heart disease. J Thorac Cardiovasc Surg. 2012;143:1264-1270.

3. Miller SP, McQuillen PS, Hamrick S, Xu D, Glidden DV, Charlton N, et al. Abnormal brain development in newborns with congenital heart disease. N Engl J Med. 2007;357:1928-1938.

4. Beca J, Gunn J, Coleman L, Hope A, Whelan LC, Gentles T, et al. Pre-operative brain injury in newborn infants with transposition of the great arteries occurs at rates similar to other complex congenital heart disease and is not related to ballon atrial septostomy. J Am Coll Cardiol. 2009:53:1807-1811.

5. Dimitropoulos A, Mcquillen PS, Sethi V, Moosa A, Chau V, Xu D, et al. Brain injury and development in newborns with critical congenital heart disease. Neurology. 2013;81:241-248.

6. Curley G, Kavanagh BP, Laffey JG. Hypocapnia and the injured brain: more harm than benefit. Crit Care Med. 2010;38:1348-1359.

7. Ulus AT, Aksoyek A, Ozkan M, Katircioglu S, Basu S. Cardiopulmonary bypass as a cause of free radical-induced oxidative stress and enhanced blood-borne isoprostanes in humans. Free Radic Biol Med. 2003;34:911-917.

8. Sanders RD, Hassell J, Davidson AJ, Robertson NJ, Ma D. Impact of anaesthetics and surgery on neurodevelopment: an update. $\mathrm{Br} \mathrm{J}$ Anaesth. $2013 ; 110: i 53-i 72$

9. Mercado-Arellano JA, Rebolledo-Ramírez J, Feria-Kaiser C, Rodríguez-Cueto G, Jasso-Gutiérrez L, García HJ. Cambios en la hemodinamia cerebral y sistémica en recién nacidos bajo sedación y analgesia con fentanilo. Bol Med Hosp Infant Mex. 1998;55:138-144.

10. Drudy PP, Gunn A, Bennet L, Ganeshaligham A, Finucane K, Buckley D, et al. Deep hypothermic circulatory arrest during arterial switch operation is associated with reduction in cerebral oxygen extraction but not increase in white matter injury. J Thorac Cardiovasc Surg. 2013;146:1327-1333.

11. O'Brien NF, Hall MW. Extracorporeal membrane oxygenation and cerebral flow velocity in children. Pediatr Crit Care Med. 2013:14:e126-e134.

12. Raju T. Cerebral Doppler studies in the fetus and the newborn infant. J Pediatr. 1991;119:165-174

13. Raju TN, Kim SY. Cerebral artery flow velocity aceleration and deceleration characteristics in newborn infants. Pediatr Res. 1989;26:588-592.

14. Nishimaki S, Iwasaki S, Minamisawa S, Seki K, Yokota S. Blood flow velocities in the anterior cerebral artery and basilar artery in asphyxiated infants. J Ultrasound Med. 2008;27:955-960.

15. Cheng HH, Wypij D, Laussen P, Bellinger D, Stopp C, Soul J, et al. Cerebral blood flow velocity and neurodevelopmental outcome in infants undergoing for congenital heart disease. Ann Thorac Surg. 2014; 98:125-132.

16. Hayashi T, Ichiyama T, Auchida M, Tashiro N, Tanaka H. Evaluation by colour Doppler and pulsed Doppler sonography of blood flow velocities in intracranial arteries during the early neonatal period. Eur J Pediatr. 1992;151:461-465 
17. Jenkins KJ, Grauvreau K, Newburger J, Spray T, Moller J, lezzoni L. Consensus-based method for risk adjustment for surgery for congenital heart disease. J Thorac Cardiovasc Surg. 2002;123:10-18.

18. Samir K, Riberi A, Ghez O, Ali M, Metras D, Kreitmann B. Delayed sternal closure: a life-saving measure in neonatal open heart surgery; could it be predictable? Eur J Cardiothorac Surg. 2002;21:787-793.

19. Zeng S, Zhou J, Peng Q, Tian L, Xu G, Zhao Y, et al. Assessment by three-dimensional power Doppler ultrasound of cerebral blood flow perfusion in fetuses with congenital heart disease. Ultrasound Obstet Gynecol. 2015;45:649-656.

20. Lynch JM, Buckley EM, Schwab PJ, McCarthy AL, Winters ME Busch DR, et al. Time-to-surgery and pre-operative cerebral hemodynamics predict post-operative with matter injury in neonates with hypoplastic left heart syndrome. J Thorac Cardiovasc Surg. 2014; $148: 2181-2188$.
21. Ilves $P$, Lintrop M, Talvik I, Muug K, Maipuu L. Changes in cerebral and visceral blood flow velocities in asphyxiated term neonates with hypoxic-ischemic encephalopathy. J Ultrasound Med. 2009;28:1471-1480.

22. Dent CL, Spaeth JP, Jones BV, Schwartz SM, Glausser TA, Hallian B, et al. Brain magnetic resonance imaging abnormalities after the Norwood procedure using regional cerebral perfusion. J Thorac Cardiovasc Surg. 2006;131:190-197.

23. Benders MJ, Hendrikse J, de Vries L, Groenendaal F, van Bel F. Doppler-assessed cerebral blood flow velocity in the neonate as estimator of global cerebral blood volume flow measured using phase-contrast magnetic resonance angiography. Neonatology. 2013;103:21-26.

24. Kudrevičienè A, Basevičius A, Lukoševičius S, Laurynaitienè J, Marmiené V, Nedzelskienè I, et al. The value of ultrasonography and Doppler sonography in prognosticating long-term outcomes among full-term newborns with perinatal asphyxia. Medicina (Kaunas). 2014;50:100-110. 